\title{
NOUVELle
}

\section{Augmentation du volume temporal chez les sujets asymptomatiques ayant des dépôts amyloïdes}

Gael Chételat
Inserm U923, École pratique des Hautes Études, Université de Caen/Basse-Normandie, Groupement d'intérêt public Cycéron, Centre hospitalier universitaire Côte de Nacre, boulevard Henri Becquerel, BP 5229, 14074 Caen, France. chetelat@cyceron.fr
> Les liens entre les dépôts amyloïdes, une des lésions caractéristiques de la maladie d'Alzheimer, et la perte neuronale soustendant les déficits cognitifs progressifs observés au cours de cette maladie dégénérative sont encore mal compris. Il est curieux par exemple que certains sujets puissent présenter une charge importante en dépôts amyloïdes sans pour autant avoir de déficits cognitifs [1]. Les résultats des quelques études ayant exploré les liens entre dépôts amyloïdes et atrophie cérébrale in vivo sont divergents [2-5]. Cette divergence pourrait s'expliquer notamment par le fait que ces liens diffèrent en fonction du degré d'évolution de la maladie ou du groupe clinique dans lequel ils sont étudiés. Nous avons montré par exemple qu'une corrélation entre atrophie et dépôts amyloïdes était observée uniquement chez des sujets âgés présentant une plainte mnésique mais aucun trouble cognitif ( $\mathrm{SCl}$ pour subjective cognitive impairment). Cette corrélation n'était retrouvée ni chez les sujets contrôles sans plainte mnésique, ni chez des patients atteints de maladie d'Alzheimer ou de mild cognitive impairment $(\mathrm{MCl})$ ayant des déficits mnésiques isolés et un risque accru de développer la maladie d'Alzheimer [6].

Dans une étude publiée en 2010 dans la revue Brain [7], nous avons cherché à identifier les différences, notamment en termes de volume cérébral régional, chez des sujets sans déficits cognitifs objectifs (contrôles et $\mathrm{SCl}$ ) entre ceux qui ont ou non des dépôts amyloïdes, et chez des sujets ayant des dépôts amyloïdes, selon qu'ils avaient ou non un déficit cognitif objectif ou subjectif ( $\mathrm{SCl}, \mathrm{MCl}$, et $\mathrm{MA}$ ).

\section{Schéma expérimental}

Tous les sujets inclus dans cette étude ont bénéficié d'un examen IRM (imagerie à résonance magnétique) pour mesurer l'atrophie (qui reflète la perte neuronale), et d'un examen PET (positron emission tomography) utilisant le Pittsburg compound $B(\mathrm{Pi})$, un radiotraceur qui permet de mesurer la charge en dépôts amyloïdes. Les participants ont été classés $\mathrm{PiB}$-positifs $(\mathrm{PiB}+)$ ou négatifs ( $\mathrm{PiB}-$ ) en fonction de la valeur PiB moyenne dans l'ensemble du cortex (divisée par la valeur de PiB dans le cervelet) et en utilisant un seuil de 1,4 déterminé par une analyse de clusters et en accord avec les études antérieures. Six groupes ont ainsi été constitués, comprenant 31 sujets contrôles $\mathrm{PiB}-$, 13 contrôles $\mathrm{PiB}+, 30 \mathrm{SCl} \mathrm{PiB}_{-}, 19 \mathrm{SCl}$ $\mathrm{PiB}+, 22 \mathrm{MCl} \mathrm{PiB}+$ et 34 patients ayant une maladie d'Alzheimer PiB+. Les données des sujets $P i B+$ ont été comparées à celles des sujets $\mathrm{PiB}$ - parmi les sujets contrôles et ayant un $\mathrm{SCl}$, et les données des sujets contrôles $\mathrm{PiB}+$ ont également été comparées à celles des patients $\mathrm{PiB}+\mathrm{SCl}, \mathrm{MCl}$ et atteints de maladie d'Alzheimer. Les données IRM, exprimées en volume de substance grise, ont été comparées au moyen d'une technique automatique d'analyse d'image utilisée classiquement (SPM pour statistical parametric mapping).

\section{Volume du lobe temporal et présence de dépôts amyloïdes}

Les patients $\mathrm{PiB}+$ ayant un déficit cognitif (subjectif ou objectif) présentaient un profil d'atrophie classique par rapport aux contrôles $\mathrm{PiB}+$ : prédominance au niveau de l'hippocampe, extension au néocortex temporal et à d'autres régions (pariétales et frontales) en fonction du groupe. En comparant, chez les patients avec une subjective cognitive impairment, ceux ayant ou non des dépôts amyloïdes (c'est-à-dire les $\mathrm{PiB}+$ versus les PiB-), une atrophie significative était retrouvée au niveau du cortex cingulaire postérieur et antérieur et du cortex temporo-pariétal (Figure 1). De façon surprenante, chez les sujets contrôles $\mathrm{PiB}+$, le volume du lobe temporal médial (dont une partie de l'hippocampe) et latéral supérieur était, à l'inverse, plus important que celui des PiB-(Figure 1). Une série d'analyses complémentaires a été réalisée afin de vérifier la validité de ces résultats et enrichir leur interprétation. Nous avons notamment souhaité éliminer une possible interférence de certains paramètres avec ces résultats: (1) le seuil de 1,4 utilisé pour séparer les sujets $\mathrm{PiB}+$ et PiB- ; (2) des différences d'âge, de niveau d'étude, de génotype de l'apolipoprotéine $\varepsilon$ (un facteur de risque de la maladie d'Alzheimer), de sexe ou de performances cognitives globales (mesurées au moyen du mini mental state examination - MMSE) entre les groupes $\mathrm{PiB}+$ et PiB- ; (3) l'utilisation de méthodes d'analyses automatiques qui peuvent induire des artéfacts liés à la transformation des images; enfin (4) l'influence de l'atrophie sur les mesures PET en raison d'effets dits de volume partiel, qui correspondent à une imprécision des mesures liée à la faible résolution spatiale des images. 
A Groupe contrôle : volume des régions cérébrales $\mathrm{PiB}+>$ PIB-
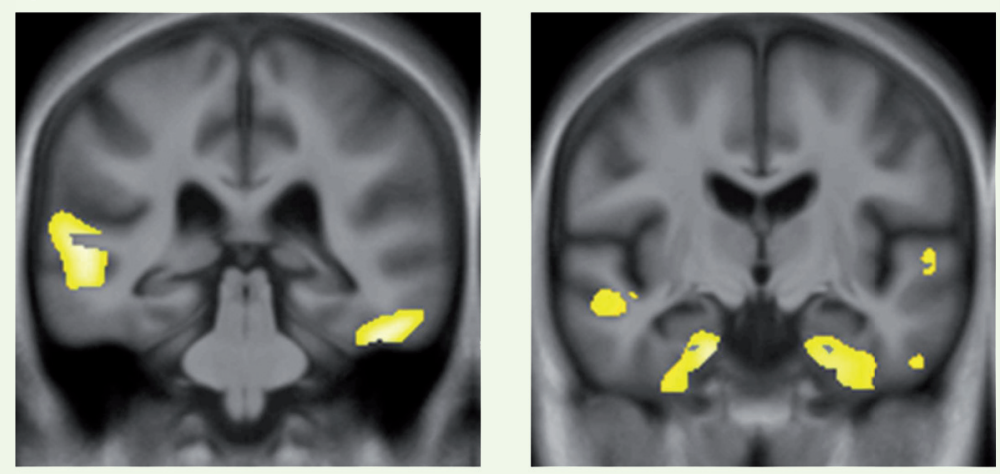

B Groupe $\mathrm{SCl}$ : volume des régions cérébrales $\mathrm{PiB}+<\mathrm{PIB}-$
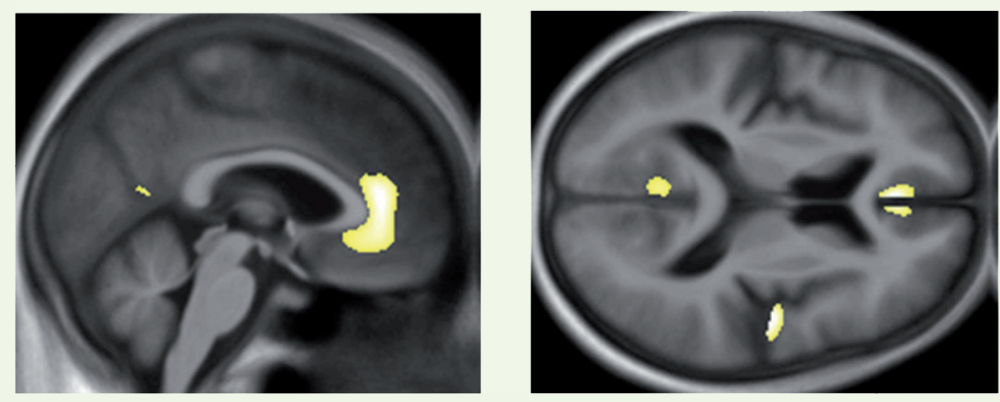

Figure 1. Localisation des modifications de volume de régions cérébrales chez les individus contrôles PiB+ et PiB- et chez les sujets ayant subjective cognitive impairment ( $\mathrm{SCl}$ ). Ces résultats ont été obtenus au moyen d'une technique automatique classique de comparaison voxel-à-voxel d'images IRM (SPM pour statistical parametric mapping). A. Les taches jaunes indiquent la localisation des régions qui sont le siège d'une augmentation de volume chez les individus contrôles $\mathrm{PiB}+$ par rapport aux individus PiB-. B. À l'inverse, les taches colorées indiquent les régions qui sont le siège d'une diminution de volume chez les patients $\mathrm{SCl} \mathrm{PiB}+$ par rapport aux patients $\mathrm{SCl}$ PiB-. Deux incidences d'IRM sont indiquées.
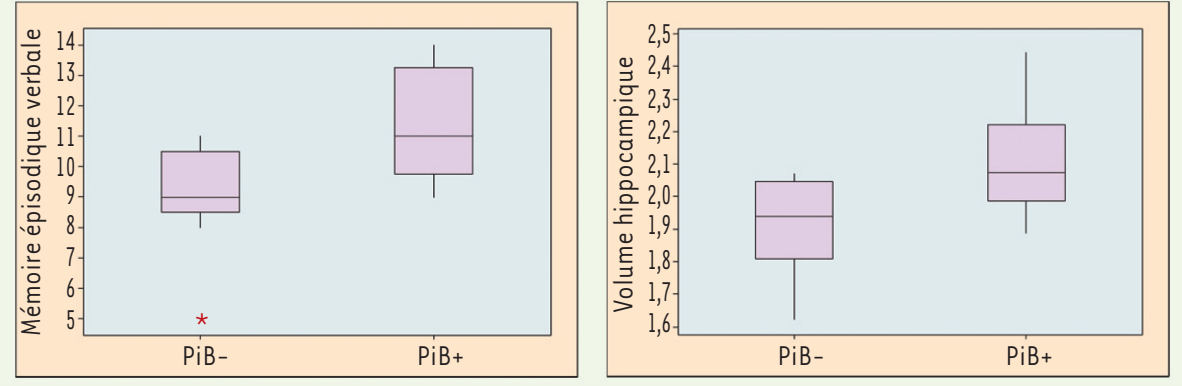

Figure 2. Comparaisons des performances mnésiques et du volume hippocampique des sujets contrôles PiB+ et PiB-. Ces données sont issues des analyses complémentaires réalisées sur un sous-échantillon de sujets appariés pour l'âge, le sexe, le niveau culturel, le génotype de l'apolipoprotéine $\varepsilon$ et le mini mental state examination. Le volume de I'hippocampe est obtenu en délimitant les contours de cette structure sur des coupes coronales de l'IRM de chacun des sujets, et les performances mnésiques correspondent au score au rappel libre différé du california verbal learning test. Ces analyses montrent que, de façon contre-intuitive, les sujets contrôles PiB+ présentent de meilleures performances et un volume hippocampique plus important que les sujets contrôles PiB-.

Nous avons donc renouvelé les analyses en utilisant des sous-groupes de sujets contrôles $\mathrm{PiB}$ + et $\mathrm{PiB}$ - définis à partir de mesures PiB corrigées pour les effets de volume partiel et en excluant les sujets ayant des valeurs PiB intermédiaires (c'est-à-dire proches du seuil de 1,4). Chaque sujet contrôle PiB était apparié selon l'âge, le sexe, le niveau culturel, le génotype de l'apolipoprotéine $\varepsilon$ et le MMSE à un sujet contrôle PiB-. De plus, afin de comparer le volume de I'hippocampe des sujets $\mathrm{PiB}+$ et $\mathrm{PiB}-$, les contours de cette structure ont été délimités manuellement plutôt qu'avec la technique automatique SPM. Les mêmes résultats étaient obtenus dans ces conditions : le volume de l'hippocampe était à nouveau supérieur chez les contrôles $\mathrm{PiB}+$ par rapport aux $\mathrm{PiB}$ (Figure 2). De plus, les performances mnésiques des contrôles PiB + étaient également meilleures que celles des PiB- (Figure 2). Une corrélation positive était retrouvée chez les sujets contrôles entre le volume de l'hippocampe et les performances mnésiques, suggérant que cette augmentation de volume chez les $\mathrm{PiB}+$ représentait un bénéfice et ne reflétait pas un processus pathologique comme une réaction inflammatoire liée aux dépôts amyloïdes. Enfin, il n'y avait pas d'augmentation de volume 
de substance blanche chez les PiB + par rapport aux $\mathrm{PiB}-$, suggérant que la différence de volume observée au niveau de la substance grise pouvait être attribuée aux corps cellulaires plutôt qu'aux neurones dans leur intégralité.

\section{Conclusion}

Dans l'ensemble, ces résultats suggèrent que la présence de dépôts amyloïdes associée à une atrophie conduit inévitablement à des déficits cognitifs, soit subjectifs - c'est-à-dire ressentis par le sujet mais pas (encore) détectables par des tests - soit objectifs. Seuls les sujets dont le volume temporal est plus important (soit parce que leur réserve cérébrale est plus importante, soit parce qu'ils ont développé des mécanismes de compensation en réponse à la présence de dépôts amyloïdes) n'auraient aucun trouble malgré la présence de dépôts amyloïdes. De plus, en accord avec notre précédente étude, la présence de dépôts amyloïdes serait associée à une atrophie uniquement à un stade très précoce (alors que les déficits cognitifs ne sont que subjectifs) et seulement dans des régions où les lésions amyloïdes sont les plus importantes, mais pas dans les régions les plus atrophiées aux stades précoces de la maladie d'Alzheimer [8]. $\diamond$

Increased temporal volume

in asymptomatic subjects

with amyloid deposits

\section{CONFLIT D'INTÉRÊTS}

L'auteur déclare n'avoir aucun conflit d'intérêts concernant les données publiées dans cet article.

\section{RÉFÉRENCES}

1. Crystal H, Dickson D, Fuld P, et al. Clinico-pathologic studies in dementia: nondemented subjects with pathologically confirmed Alzheimer's disease. Neurology $1988 ; 38: 1682-7$.

2. Jack CR Jr, Lowe VJ, Senjem ML, et al. 11C PiB and structural MRI provide complementary information in imaging of Alzheimer's disease and amnestic mild cognitive impairment. Brain 2008 ; $131: 665-80$.

3. Storandt M, Mintun MA, Head D, Morris JC. Cognitive decline and brain volume loss as signatures of cerebral amyloid-beta peptide deposition identified with Pittsburgh compound B: cognitive decline associated with Abeta deposition. Arch Neurol 2009 ; 66: 1476-81.

4. Dickerson BC, Bakkour A, Salat DH, et al. The cortical signature of Alzheimer's disease: regionally specific cortical thinning relates to symptom severity in very mild to mild $A D$ dementia and is detectable in asymptomatic amyloid-positive individuals. Cereb Cortex 2009 ; 19 : 497-510.

5. Bourgeat P, Chételat G, Villemagne VL, et al. Betaamyloid burden in the temporal neocortex is related to hippocampal atrophy in elderly subjects without dementia. Neurology $2010 ; 74$ : 121-7.

6. Chételat $G$, Villemagne VL, Bourgeat $P$, et al. Relationship between atrophy and $\beta$-amyloid deposition in Alzheimer's disease. Ann Neurol 2010 ; $67: 317-24$.

7. Chételat G, Villemagne VL, Pike KE, et al. Larger temporal volume in asymptomatic elderly with high versus low beta-amyloid deposition. Brain 2010 ; 133 : 3349-58.

8. Chételat $\mathrm{G}$, Villemagne VL, Bourgeat $P$, et al. Relationship between atrophy and $\beta$-amyloid deposition in Alzheimer's disease. Ann Neurol 2010 ; $67: 317-24$.

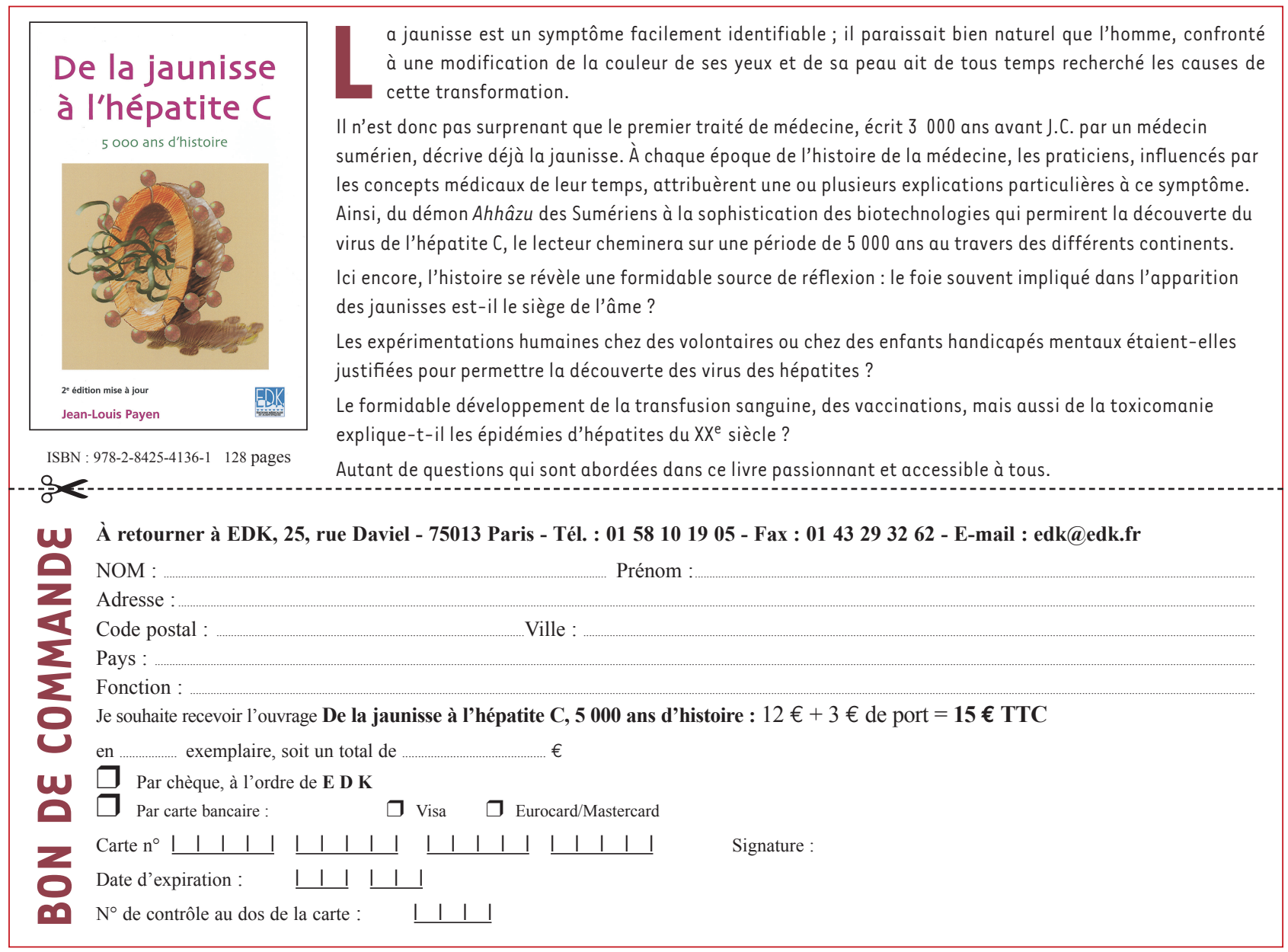

